\title{
NETS OF EXTREME BANACH LIMITS
}

\author{
RODNEY NILLSEN
}

\begin{abstract}
Let $N$ be the set of natural numbers and let $\sigma: N \rightarrow N$ be an injection having no periodic points. Let $M_{\sigma}$ be the set of $\sigma$-invariant means on $l_{\infty}$. When $f \in l_{\infty}$ let $\bar{d}_{\sigma}(f)=\sup \lambda(f)$, where the supremum is taken over all $\lambda \in M_{\sigma}$. It is shown that when $f \in l_{\infty}$, there is a sequence $\left(\lambda_{s}\right)_{s=2}^{\infty}$ of extreme points of $M_{\sigma}$ which has no extreme weak* limit points and such that $\lambda_{s}(f)=\bar{d}_{\sigma}(f)$ for $s=2,3, \ldots$ As a consequence, the extreme points of $M_{\sigma}$ are not weak* compact.
\end{abstract}

1. Introduction. Let $N$ denote the set of natural numbers, including 0 . Let $l_{\infty}$ be all bounded real valued functions on $N$ and let $l_{\infty}^{\prime}$ be its dual. A mean is an element $\mu \in l_{\infty}^{\prime}$ such that $\|\mu\|=\mu(1)=1$. Let $M$ be the set of all means. Let $\sigma: N \rightarrow N$ be an injection having no periodic points. Such a map will be called a motion. The motion $n \rightarrow n+1$ will be denoted by $\tau$. A mean $\mu$ is said to be $\sigma$-invariant if $\mu(f \circ \sigma)=\mu(f)$ for all $f \in l_{\infty}$. Let $M_{\sigma}$ be the set of all $\sigma$ invariant means. Both $M$ and $M_{\sigma}$ are weak ${ }^{*}$ compact convex sets. $M_{\tau}$ is known as the set of Banach limits. If $\lambda \in M_{\sigma}$ and $f \in l_{\infty}$ it is shown in this paper that there is a sequence $\left(\lambda_{s}\right)_{s=2}^{\infty}$ of extreme points of $M_{\sigma}$ so that no limit point of this sequence is extreme and $\lambda_{s}(f) \geqslant \lambda(f)$ for all $s$. This implies that $M_{\sigma}$ has a non-weak* compact set of extreme points. An application is given to deducing a result of Raimi concerning nonequicontinuity of certain homeomorphisms on the Stone-Čech compactification $\beta N$ of $N$.

2. Preliminary results. Let $N^{*}$ be the set of nonzero natural numbers. If $m \in N$ and $n \in N^{*}$ we define $\mu_{\sigma}(m, n) \in M$ by

$$
\mu_{\sigma}(m, n)(f)=(1 / n)\left(\sum_{k=0}^{n-1} f\left(\sigma^{k} m\right)\right) \text { for } f \in l_{\infty} .
$$

The following lemma is then well known (see [7] for a proof).

LEMMA 2.1. Let I be a directed set and let $\left(\mu_{\sigma}\left(m_{\alpha}, n_{\alpha}\right)\right)_{\alpha \in I}$ be a net of means each of the form (2.1). Then if $\lim _{\alpha} n_{\alpha}=+\infty$, any weak limit point of $\left(\mu_{\sigma}\left(m_{\alpha}, n_{\alpha}\right)\right)_{\alpha \in I}$ belongs to $M_{\sigma}$.

If $A \subseteq N$ let $\chi_{A}$ be the characteristic function of $A$. When $\mu \in M$ we shall write $\mu(A)$ instead of $\mu\left(\chi_{A}\right)$. If $m \in N$ and $n \in N^{*}$ let $I_{\sigma}(m, n)=\left\{\sigma^{i} m: i\right.$ $=0,1, \ldots, n-1\}$. When $B \subseteq N$ is finite let $|B|$ be the cardinality of $B$. By (2.1) we have

Received by the editors March 3, 1975.

AMS (MOS) subject classifications (1970). Primary 28A70, 43A07.

Key words and phrases. Motions, means, invariant means, Banach limits, extreme points, ergodic measure, Stone-Čech compactification. 


$$
\mu_{\sigma}(m, n)(A)=(1 / n)\left(\left|A \cap I_{\sigma}(m, n)\right|\right) .
$$

We let, for $f \in l_{\infty}$,

$$
\bar{d}_{\sigma}(f)=\sup _{\lambda \in M_{\sigma}} \lambda(f) \text { and } \underline{d}_{\sigma}(f)=\inf _{\lambda \in M_{\sigma}} \lambda(f) .
$$

When $A \subseteq N$ we write $\bar{d}_{\sigma}(A), \underline{d}_{\sigma}(A)$ respectively in place of $\bar{d}_{\sigma}\left(\chi_{A}\right), \underline{d}_{\sigma}\left(\chi_{A}\right)$.

LEMMA 2.2. For $f \in l_{\infty}$ we have

$$
\bar{d}_{\sigma}(f)=\limsup _{n \rightarrow \infty}\left(\limsup _{m \rightarrow \infty} \mu_{\sigma}(m, n)(f)\right)
$$

and

$$
\underline{d}_{\sigma}(f)=\liminf _{n \rightarrow \infty}\left(\liminf _{m \rightarrow \infty} \mu_{\sigma}(m, n)(f)\right) .
$$

Proof. Let $T: l_{\infty} \rightarrow l_{\infty}$ be the linear map $f \rightarrow f \circ \sigma$. In matrix terms $T$ arises from multiplication by the matrix $A=\left(\alpha_{i j}\right)$, where for $i, j \in N, \alpha_{i j}=0$ if $j \neq \sigma(i)$ and $\alpha_{i j}=1$ if $j=\sigma(i)$. If we let

$$
A_{n}=(1 / n)\left(I+A+A^{2}+\cdots+A^{n-1}\right),
$$

where $I$ is the infinite identity matrix, then (3.2.1) and (3.2.2) of [4] hold, where $\mathcal{Q}=\left\{A^{n}: n \in N\right\}$ and $\left\{A_{\alpha}\right\}$ is the net $\left(A_{n}\right)_{n \in N}$. The result now follows from Corollary 3.7 of [4].

The following lemma now follows easily from (2.4).

LemmA 2.3. Let $f \in l_{\infty}$. Then there are sequences $\left(p_{n}\right)$ and $\left(q_{n}\right)$ in $N^{*}$ so that

(2.6) $\lim _{n \rightarrow \infty} q_{n}=+\infty$,

(2.7) $\lim _{n \rightarrow \infty} \mu_{\tau}\left(p_{n}, q_{n}\right)(f)=\bar{d}_{\tau}(f)$, and

(2.8) the sets $\left.I_{\tau}\left(p_{n}, q_{n}\right)\right)_{n \in N}$ are pairwise disjoint.

Lemma 2.4. Let $\sigma_{1}$ and $\sigma_{2}$ be two motions on $N$. Suppose that $Z \subseteq N$ is such that $\bar{d}_{\sigma_{1}}(Z)=0$ and $\sigma_{1}=\sigma_{2}$ on $N-Z$. Then $M_{\sigma_{1}} \subseteq M_{\sigma_{2}}$.

Proof. Let $f \in l_{\infty}$ and $\mu \in M_{\sigma_{1}}$. Then $\mu(N-Z)=1$ so that $\mu\left(f \circ \sigma_{2}\right)$ $=\mu\left(\left(f \circ \sigma_{2}\right) \chi_{N-Z}\right)=\mu\left(\left(f \circ \sigma_{1}\right) \chi_{N-Z}\right)=\mu\left(f \circ \sigma_{1}\right)=\mu(f)$. Hence $\mu \in M_{\sigma_{2}}$.

3. Nets of extreme points of $M_{\sigma}$. First we shall consider the case when $\sigma=\tau$.

THEOREM 3.1. Let $f \in l_{\infty}$. Then there is a net $\left(\lambda_{s}\right)_{s=2}^{\infty}$ of extreme points of $M_{\tau}$ so that no limit point of this net is extreme and $\lambda_{s}(f)=\bar{d}_{\tau}(f)$ for each $s$.

Proof. The proof is divided into a number of steps.

Step I. By Lemma 2.3 we can find sequences $p_{11}, p_{21}, p_{22}, p_{31}, p_{32}, p_{33}, p_{41}$, $\ldots$ and $q_{11}, q_{21}, q_{22}, q_{31}, q_{32}, q_{33}, q_{41}, \ldots$ in $N^{*}$ so that if we let $\lambda_{r s}$ $=\mu_{\tau}\left(p_{r s}, q_{r s}\right)$ then

$$
\begin{aligned}
\lim _{r \rightarrow \infty} q_{r s} & =+\infty \text { for each } s, \\
\lim _{r \rightarrow \infty} \lambda_{r s}(f) & =\bar{d}_{\tau}(f) \text { for each } s, \text { and }
\end{aligned}
$$


(3.3) the sets $\left(I_{\tau}\left(p_{r s}, q_{r s}\right)\right)_{r \geqslant s}$ are pairwise disjoint. Let us write $q_{r s}$ $=t_{r s}(2 s+1)+u_{r s}$ where $t_{r s}, u_{r s} \in N$ and $0 \leqslant u_{r s}<(2 s+1)$.

By (2.6) there is no loss of generality in assuming that $t_{r s} \geqslant 1$ so that by (3.1) we have for each $s$

$$
t_{r s} \geqslant 1, \lim _{r \rightarrow \infty} q_{r s} / t_{r s}(2 s+1)=1 \text { and } \lim _{r \rightarrow \infty} u_{r s} / q_{r s}=0 .
$$

Step II. For $r, s \in N^{*}$ with $r \geqslant s$ let $X_{r s}=I_{\tau}\left(p_{r s}, t_{r s}(2 s+1)\right)$. By (3.3) the sets $\left(X_{r s}\right)_{r \geqslant s}$ are pairwise disjoint. For $s \in N^{*}$ let $B_{s}=\{0, s, s+1, \ldots, 2 s$ $-1\}$, let

$$
B_{r s}=\bigcup_{k=0}^{t_{r s}-1}\left(k(2 s+1)+B_{s}\right)
$$

and let

$$
B=\bigcup_{r=1}^{\infty}\left(\bigcup_{s=1}^{r}\left(p_{r s}+B_{r s}\right)\right)
$$

Then we have

$$
\left(p_{r s}+B_{r s}\right) \cup\left\{p_{r s}+t_{r s}(2 s+1)-1\right\} \subseteq X_{r s}
$$

and

$$
(B \triangle \tau B) \cap\left(p_{r s}+B_{r s}\right)=p_{r s}+\left(B_{r s} \triangle \tau B_{r s}\right)
$$

so that, when $r \geqslant s \geqslant 2$,

$$
(B \triangle \tau B) \cap\left(p_{r s}+B_{r s}\right)=p_{r s}+\bigcup_{k=0}^{t_{r s}-1}(k(2 s+1)+\{0,1, s, 2 s\}) .
$$

Now let $\mu_{r s}=\mu_{\tau}\left(p_{r s}, t_{r s}(2 s+1)\right)$. Then $\mu_{r s} \in M$ and using (3.4) and (3.2) we have

$$
\lim _{r \rightarrow \infty} \mu_{r s}(f)=\bar{d}_{\tau}(f) \text { for each } s \in N^{*} .
$$

From the definitions of $X_{r s}$ and $\mu_{r s}$ and (2.2) we have

$$
\mu_{r s}\left(X_{r s}\right)=1 \text {, when } r \geqslant s \text {. }
$$

Step III. For $s \in N, s \geqslant 2$ we let $X_{s}=\cup_{r=s}^{\infty} X_{r s}$. Writing $X_{s}=\left\{m_{1}\right.$, $\left.m_{2}, \ldots\right\}$ and $N-X_{s}=\left\{n_{1}, n_{2}, \ldots\right\}$ where $m_{1}<m_{2}<\cdots$ and $n_{1}<n_{2}$ $<\cdots$ we can define a motion $\tau_{s}$ on $N$ by letting $\tau_{s}\left(m_{i}\right)=m_{i+1}$ and $\tau_{s}\left(n_{i}\right)=n_{i+1}$. Then on $X_{s}, \tau_{s}$ acts in the way that $\tau$ acts on $N$ so that by using (2.4), (2.5), (3.6), (3.7), (3.8) we see that if $\lambda \in M_{\tau_{s}}$ and $\lambda\left(X_{s}\right)=1$ then $\lambda(B)=(s+1) /(2 s+1)$ and $\lambda(B \triangle \tau B)=4 /(2 s+1)$. Let

$$
Z_{s}=\bigcup_{r=s}^{\infty}\left\{p_{r s}-1, p_{r s}+t_{r s}(2 s+1)-1\right\}
$$

Then $\tau=\tau_{s}$ on $N-Z_{s}$ and by $(2.4) \bar{d}_{\tau}\left(Z_{s}\right)=0$. Using Lemma 2.4 we see that

$$
\begin{aligned}
& \text { if } \lambda \in M_{\tau} \text { and } \lambda\left(X_{s}\right)=1 \text { then } \lambda(B)=(s+1) /(2 s+1) \text { and } \\
& \lambda(B \triangle \tau B)=4 /(2 s+1) \text {. }
\end{aligned}
$$


Step IV. When $s \geqslant 2$ let $\mu_{s}$ be a limit point of the net $\left(\mu_{r s}\right)_{r=s}^{\infty}$. By Lemma 2.1, (3.1), (3.9) and (3.10) we have $\mu_{s} \in M_{\tau}, \mu_{s}(f)=\bar{d}_{\tau}(f)$ and $\mu_{s}\left(X_{s}\right)=1$. If we let $L_{s}=\left\{\lambda: \lambda \in M_{\tau}, \lambda\left(X_{s}\right)=1\right.$ and $\left.\lambda(f)=\bar{d}_{\tau}(f)\right\}$, this shows that $L_{s}$ is not void. $L_{s}$ is a weak* compact convex set and so has extreme points. Also if $\lambda_{1}, \lambda_{2} \in M_{\tau}$ and $0 \leqslant \alpha \leqslant 1$, from (2.3) it follows that if $\alpha \lambda_{1}+(1-\alpha) \lambda_{2}$ $\in L_{s}$ then $\lambda_{1}, \lambda_{2} \in L_{s}$. Hence any extreme point of $L_{s}$ is an extreme point of $M_{\tau}$ so that there is an extreme point $\lambda_{s}$ of $M_{\tau}$ with $\lambda_{s}\left(X_{s}\right)=1$ and $\lambda_{s}(f)$ $=\bar{d}_{\tau}(f)$. By $(3.11) \lambda_{s}(B)=(s+1) /(2 s+1)$ and $\lambda_{s}(B \triangle \tau B)=4 /(2 s+1)$. Hence if $\lambda$ is a limit point of the net $\left(\lambda_{s}\right)_{s=2}^{\infty}$ we will have $\lambda(B)=\frac{1}{2}$ and $\lambda(B \triangle \tau B)=0$. By Proposition 10.4 of [9], $\lambda$ is not an extreme point of $M_{\tau}$. Hence $\left(\lambda_{s}\right)_{s=2}^{\infty}$ is a net of extreme points of $M_{\tau}$ which has the required properties.

THEOREM 3.2. Let $f \in l_{\infty}$. Then there is a net $\left(\nu_{s}\right)_{s=2}^{\infty}$ of extreme points of $M_{\sigma}$ so that no limit point of this net is extreme and $\nu_{s}(f)=\bar{d}_{\sigma}(f)$ for each $s$.

Proof. By Theorem 4.8 of [3] we may assume that there is $m \in N$ for which $\left\{\sigma^{i} m: i \in N\right\}=N$, because $\sigma$ can if necessary be replaced by another notion $\mu$ for which this is the case, and with $M_{\sigma}=M_{\mu}$. Consider the map $\psi: i \rightarrow \sigma^{i} m$ from $N$ into $N$. The map on $l_{\infty}$ given by $h \rightarrow h \circ \psi$ induces a dual map $S$ on $l_{\infty}^{\prime}$. Because $\sigma \circ \psi=\psi \circ \tau$ on $N$ we find that $S$ maps $M_{\tau}$ onto $M_{\sigma} . S$ is a weak* continuous, affine bijection from $M_{\tau}$ onto $M_{\sigma}$. Let $g=f \circ \psi$. Then $\bar{d}_{\tau}(g)$ $=\bar{d}_{\sigma}(f)$. By Theorem 3.1 we can choose a net $\left(\lambda_{s}\right)_{s=2}^{\infty}$ of extreme points of $M_{\tau}$ so that no limit point of this net is extreme and $\lambda_{s}(g)=\bar{d}_{\tau}(g)$ for each $s$. We let $\nu_{s}=S \lambda_{s}$. Then $\left(\nu_{s}\right)_{s=2}^{\infty}$ is a net of extreme points of $M_{\sigma}$, no limit point of which is extreme. Also $\nu_{s}(f)=\lambda_{s}(g)=\bar{d}_{\tau}(g)=\bar{d}_{\sigma}(f)$ for all $s$.

\section{COROllary 3.3. The set of extreme points of $M_{\sigma}$ is not weak* compact.}

A motion $\sigma$ on $N$ can be extended to a homeomorphism (which we also denote by $\sigma$ ) from $\beta N$ into $\beta N$. If $\lambda \in M_{\sigma}, \lambda$ given rise to a measure (which we also denote by $\lambda$ ) on $\beta N$ so that $\lambda(f)=\int \bar{f} d \lambda$ for all $f \in l_{\infty}$, where $\bar{f}$ is the continuous extension of $f$ to $\beta N$. When $\lambda \in M_{\sigma}$, the support of $\lambda$ is a $\sigma$ invariant subset of $\beta N-N$. It has been shown in [1] that there is a set $A \subseteq N$ such that $\bar{d}_{\sigma}(A)>0$ but $\lambda(A)=0$ for all $\lambda \in M_{\sigma}$ such that $\lambda$ has a support which is a $\sigma$-minimal set. Hence we have

COROllary 3.4. There is a net $\left(\lambda_{s}\right)_{s=2}^{\infty}$ of ergodic $\sigma$-invariant measures on $\beta N-N$ such that each $\lambda_{s}$ has a non-o-minimal support and no limit point of this net is ergodic.

If $A \subseteq N$ and $\lambda(A)=1$ for some $\lambda \in M_{\sigma}$ then (support of $\lambda$ ) $\subseteq \bar{A}$ $\cap(\beta N-N)$. Since the support of $\lambda$ is closed and $\sigma$-invariant it contains a $\sigma$ minimal set, so there is an extreme point $\nu$ of $M_{\sigma}$ having $\sigma$-minimal support and $\nu(A)=1$. This fact can be used in Step IV of the proof of Theorem 3.1 as follows. Let $f=1 \in l_{\infty}$ and recall that $\mu_{s} \in M_{\tau}$ and $\mu_{s}\left(X_{s}\right)=1$. Then there is an extreme point $\lambda_{s}$ of $M_{\tau}$ so that $\lambda_{s}\left(X_{s}\right)=1$ and $\lambda_{s}$ has $\tau$-minimal support. If $s_{1} \neq s_{2}$ the supports of $\lambda_{s_{1}}$ and $\lambda_{s_{2}}$ are disjoint since $\lambda_{s_{1}}\left(X_{s_{1}}\right)=1$, $\lambda_{s_{2}}\left(X_{s_{2}}\right)=1$ and $X_{s_{1}} \cap X_{s_{2}}=\varnothing$ from the definition of $X_{s}$ and (3.3). Given a motion $\sigma$, let $S$ be as defined in the proof of Theorem 3.2. Then if $\nu_{s}=S \lambda_{s}, \nu_{s}$ is an extreme point of $M_{\sigma}$, the support of $\nu_{s}$ is $\sigma$-minimal and since no limit 
point of $\left(\lambda_{s}\right)_{s=2}^{\infty}$ is extreme in $M_{\tau}$, no limit point of $\left(\nu_{s}\right)_{s=2}^{\infty}$ is extreme in $\boldsymbol{M}_{\sigma}$. Because the $\lambda_{s}$ have pairwise disjoint supports, this is also true of the $\nu_{s}$. Hence we have proved

COROllaRY 3.5. There is a net $\left(\nu_{s}\right)_{s=2}^{\infty}$ of ergodic o-invariant measures on $\beta N-N$ such that the supports of the $\nu_{s}$ are pairwise disjoint and $\sigma$-minimal and no limit point of this net is ergodic.

The following result is Theorem 3.2 in Raimi's paper [11].

COROllary 3.6. The family $\left\{\sigma^{n}: n \in N\right\}$ is not an equicontinuous family of homeomorphisms on $\beta N-N$.

Proof. Were $\left\{\sigma^{n}: n \in N\right\}$ an equicontinuous family on $\beta N-N, M_{\sigma}$ would have a weak* compact set of extreme points by Theorem 4.5 of [2]. By Corollary 3.3 this is not the case.

4. Remarks. (1) A point $\omega \in \beta N-N$ is said to be $\sigma$-discrete if the set $O_{\sigma}(\omega)=\left\{\sigma^{i} \omega: i \in N\right\}$ is discrete in $\beta N-N$. It is shown in Proposition 1.2 of [1] that $\beta N-N$ has a dense set of $\sigma$-discrete points. If we let $A^{\sigma}$ be the union of all $\sigma$-minimal sets in $\beta N-N$ and let $K^{\sigma}$ be the union of all supports of elements of $M_{\sigma}$, it is shown in [8] that both $\overline{A^{\sigma}}$ and $\overline{K^{\sigma}}$ have dense sets of $\sigma$ discrete points. If $\omega$ is $\sigma$-discrete then $\overline{O_{\sigma}(\omega)}$ is homeomorphic to $\beta N$ by extending the map $i \rightarrow \sigma^{i} \omega$ on $N$ continuously to $\beta N$. Then by applying Theorem 3.2 and using the results of [1] and [8] many other examples of nets of extreme points of $M_{\sigma}$ having no extreme limit points can be constructed.

(2) The example on p. 383 of [2] seems to indicate that Raimi's result in Corollary 3.6 does not readily imply Theorem 3.2 .

(3) In Theorem 3.4 of [11] Raimi proves that no homeomorphism $h$ of $\beta N-N$ has an equicontinuous family of iterates on any infinite $h$-minimal set. This is a stronger result than Corollary 3.6 above and the basic construction of this paper seems inadequate to deduce this fact, even when $h$ is a motion on $\beta N-N$. If a net $\left(\nu_{s}\right)_{s=2}^{\infty}$ of extreme points of $M_{\sigma}$ could be constructed so that no limit point was extreme but the measures $\nu_{s}$ had a common $\sigma$-minimal support (compare this with Corollary 3.5) then Theorem 3.4 of [11] would follow for motions of $\beta N-N$ by using 4.5 of [2].

(4) The results of Raimi mentioned above have been generalized to $F$-spaces by Gait in [5], notably in his results $2.1,2.2$ and 4.1. While it seems that the results presented here do not imply those of Gait, it is possible that the basic construction in this paper, if carried out on a topological group $G$ instead of $N$, would imply Gait's results, in some cases. Letting $M_{G}$ be the set of invariant means on $G$ (see [6]), the idea would be to construct a net of extreme points of $M_{G}$ having no extreme limit points, and then reason as in Corollary 3.6 above.

The author would like to thank the referee for a number of helpful suggestions.

\section{REFERENCES}

1. C. Chou, Minimal sets and ergodic measures for $\beta N-N$, Illinois J. Math. 13 (1969), 777-788. MR 40 \#2814. 
2. G. Converse, I. Namioka and R. Phelps, Extreme invariant positive operators, Trans. Amer. Math. Soc. 137 (1969), 375-385. MR 39 \#4692.

3. D. Dean and R. Raimi, Permutations with comparable sets of invariant means, Duke Math. J. 27 (1960), 467-479. MR 22 \# 12397.

4. J. P. Duran, Almost convergence, summability and ergodicity, Canad. J. Math. 27 (1974), 372-387. MR 49 \#5636.

5. J. Gait, Transformation groups with no equicontinuous minimal set, Compositio Math. 25(1972), 87-92. MR 47 \#4233.

6. F. P. Greenleaf, Invariant means on topological groups, Van Nostrand, Princeton, N.J., 1969. MR 40 \#4776.

7. M. Jerison, The set of all generalized limits of bounded sequences, Canadian J. Math. 9 (1957), 79-89. MR 18, 747.

8. R. Nillsen, Discrete orbits in $\beta N-N$, Colloq. Math. 33 (1975), 71-81.

9. R. Phelps, Lectures on Choquet's theorem, Van Nostrand, Princeton, N.J., 1966. MR 33 $\# 1690$.

10. R. Raimi, Invariant means and invariant matrix methods of summability, Duke Math. J. 30 (1963), 81-94. MR 27 \#3965.

11. - Homeomorphisms and invariant measures for $\beta N-N$, Duke Math. J. 33 (1966), 1-12. MR 33 \#6608.

Department of Mathematics, University of Wollongong, Wollongong, New South Wales, Australia 